\title{
Iris Repair after Long-Term Complications of Angle-Supported Phakic Intraocular Lenses
}

\section{Camille Budo}

Ophthalmology Polyclinic Sint-Truiden, Sint-Truiden, Belgium.

Email: camille.budo@skynet.be

Received January $17^{\text {th }}, 2012$; revised February $20^{\text {th }}, 2012$; accepted March $9^{\text {th }}, 2012$

\begin{abstract}
We report the case of a high myopic patient who had been implanted with angle-supported phakic intraocular lenses (pIOL) in 1990 and who subsequently and gradually developed complications in both eyes including endothelial cell loss, chronic glaucoma, cataract, pupil ovalization and severe iris atrophy. The patient was impaired by photophobia, glare, halo, loss in visual acuity and concerned about the cosmetically deforming aspect of her eyes. Cataract surgery was performed after explantation of the pIOL followed by combined implantation of a standard IOL in the capsular bag and a Dr. Schmidt artificial iris in the sulcus. There were no intraoperative and postoperative complications except slight bilateral corneal oedema which resolved completely within 1 month after surgery. Uncorrected and corrected visual acuity improved progressively to reach 0.3 and 0.6 at the last follow-up visit ( \pm 1 year), respectively. The patient was very satisfied with the functional and aesthetical outcomes.
\end{abstract}

Keywords: Artificial Iris; Prosthetic Iris; Angle-Supported Phakic Intraocular Lens

\section{Introduction}

Patients with iris defects, either from congenital, traumatic or other causes, are often significantly incapacitated by varying amounts of visual disability including photophobia, glare, reduced visual acuity, loss of contrast sensitivity and depth of focus. In addition, patients, particularly those with large iris damages, are likely to experience substantial psychological distress from the unaesthetic appearance of their eyes. In many cases, a surgical intervention is the only treatment option to address the functional problems caused by iris damage. Simple suture repair to create a new pupillary aperture is usually the preferred approach when the quality and amount of the remaining iris tissue is sufficient to ensure a successful outcome. However, in more complex cases, an iris prosthesis is necessary.

Iris implants have been available since 1991 and many studies have shown their efficacy to resolve the visual symptoms associated with iris defects [1-6]. However, most implant models have substantial limitations; in particular most require large incisions for implantation and do not restore the original appearance of the iris. In the past few years, though, valuable progress has been made in this field with the introduction on the European market of an iris prosthesis manufactured by Dr. Schmidt Intraoculalinsen $\mathrm{GmbH} / \mathrm{HumanOptics}$ AG (Germany) which offers some features not available in the other models.
This device is a foldable prosthesis that can be inserted in the sulcus through a small incision $(\leq 3.2 \mathrm{~mm})$ and custom-made for each patient to match the patient's natural iris colour.

We report the functional and aesthetic outcomes in a patient who had a Dr. Schmidt iris prosthesis implantation in both eyes to repair an extensive iris atrophy following angle-supported phakic IOL (pIOL) implantation. Iris reconstruction was combined with pIOL explantation, cataract removal with capsular bag IOL implantation.

\section{Case Report}

A 35 year-old woman presented to the University Clinic of Maastricht in August 2002 complaining of decrease in visual acuity, photophobia and disturbing glare and halo. The patient had a history of high myopia in both eyes which was treated with anterior chamber angle-supported pIOL in 1990 (Morcher type 54; power pIOLs-22 D). A review of her medical records indicated an uncorrected distance visual acuity (UDVA) following treatment of $0.7(20 / 29)$ in the right eye and $0.8(20 / 25)$ in the left eye. Eight years later (1998), the patient had gradually developed chronic glaucoma associated with iris deformation in both eyes. Her glaucoma was controlled with topical $\beta$-blockers i.e. Timolol (Timoptol, MSD, Belgium) and Travoprost (Travatan, Alcon, Belgium).

At her first visit to the clinic in 2002, a slit lamp ex- 
amination revealed pupil ovalization and iris atrophy. The UDVA had decreased to $0.6(20 / 40)$ in the right eye $\left(-4.75-0.75 \times 72^{\circ}\right)$ and to $0.2(20 / 100)$ in the left eye $\left(-4.00-2.00 \times 156^{\circ}\right)$. Endothelial cell density was about 1100 cells $/ \mathrm{mm}^{2}$ in the right eye and 2300 cells $/ \mathrm{mm}^{2}$ in the left eye. Intraocular pressure (IOP) was $18 \mathrm{mmHg}$ in the right eye and $22 \mathrm{mmHg}$ in the left eye. Two further examinations took place until January 2003. Although the patient was informed of her serious condition, she didn't consult again until August 2009 when she complained of worsening visual symptoms and further iris deformation (see Figure 1(A)). Upon examination, UDVA was $0.5(20 / 40)$ in her right eye and $0.3(20 / 70)$ in her left eye. Endothelial cell density had remained approximately the same in the right eye $\left(1008\right.$ cells $\left./ \mathrm{mm}^{2}\right)$ but had significantly decreased in her left eye (1662 cells/ $\mathrm{mm}^{2}$ ). There was evidence of nuclear cataract and increase of IOP in both eyes (OD: $23 \mathrm{mmHg}$; OS: 27 $\mathrm{mmHg}$ ). Together we decided upon explantation of the pIOL followed by simultaneous phacoemulsification, capsular bag IOL implantation and iris reconstruction in both eyes. In this case, due to the extent of iris damage and poor quality of the remaining tissue, simple suturing was not an option and we opted for an artificial iris implant from Dr. Schmidt Intraocularlinsen. This foldable prosthesis is made of a pigmented, biocompatible silicone elastomer with a diameter of $12.80 \mathrm{~mm}$ and a fixed pupillary aperture of $3.35 \mathrm{~mm}$. It is available in two versions; one containing a polymer fiber meshwork that allows the device to be sutured, and one without the meshwork for sutureless fixation. Both versions can be folded and implanted into the ciliary sulcus through a mini incision. The device is custom-made with handcrafted adjustment of the colour to mimic patient's original iris colour. For this patient, two customized fibre-free artificial iris were manufactured based on a photograph of the patient's residual iris. The sulcus size was estimated by measuring the limbus size (white to white diameter) and by adding $0.5 \mathrm{~mm}$. Surgery was scheduled for her left eye in March 2010. The second surgery took place in September 2010.

Surgeries were performed under general anaesthesia. The surgery procedure consisted of explantation of the pIOL via a limbal incision of approximately $6.5 \mathrm{~mm}$, followed by phacoemulsification, irrigation/aspiration and implantation of an AcrySof MN60MA IOL (OS:-4D; OD:-5D) in the capsular bag (Alcon, Belgium). Calculation of the IOL power was performed using the standard SRK-T formula, and preoperative axial length and keratometry were performed using ultrasound. In the final surgical step, the iris prosthesis was inserted into the anterior chamber with a folding forceps through the same incision as that used for the pIOL explantation and the standard IOL implantation and then positioned under the natural iris remnants into the sulcus. The corneal wound was then sutured with 9-0 nylon sutures, which were removed 6 weeks after the surgery. There were no intraoperative complications. The patient was followed-up very closely during the first month and then approximately on a monthly basis thereafter. Follow-up was 14 months for the left eye and 10 months for the right eye.

Two weeks after the surgery, CDVA was 0.3 in the left eye $\left(+1.50-6.50 \times 163^{\circ}\right)$ and 0.45 in the right eye $\left(-1.50-3.00 \times 10^{\circ}\right)$. The iris prostheses were well positioned and covered the entire iris defect. There was no rise in IOP and values were within normal range (OD: 21 $\mathrm{mmHg}$; OS: $20 \mathrm{mmHg}$ ). Endothelial cell density was 1446 cells $/ \mathrm{mm}^{2}$ in the left eye and 584 cells $/ \mathrm{mm}^{2}$ in the right eye and no corneal decompensation occurred. There were no postoperative complications except a small corneal oedema in both eyes which had resolved within a month post-surgery. At the 6-month follow-up visit, CDVA had improved to $0.6(20 / 33)$ in the left eye $(-1.00$ $\left.-1.00 \times 12^{\circ}\right)$ and had remained at 0.45 in the right eye $\left(-2.50-2.75 \times 17^{\circ}\right)$. At the 10 -month visit, an IOP spike occurred in the left eye $(33 \mathrm{mmHg})$ requiring an increase in antiglaucoma medications i.e. Acetazolamide (Diamox, Goldshield Pharmaceuticals Ltd, UK), Dorzolamide/ Timolol (Cosopt, MSD, Belgium), Latanoprost (Xalatan, Pfizer, Belgium) and Brinzolamide/Timolol (Azarga, Alcon, Belgium). At the most recent follow-up visit (June 2011), the CDVA had improved to 0.6 (20/33) in the right eye and had remained stable in the left eye (0.6). IOP was normal in both eyes (OS: $17 \mathrm{mmHg}$; OD: 14 $\mathrm{mmHg}$ ). The patient was delighted with the results. Her visual symptoms had resolved and she was very satisfied with the cosmetic appearance of her eyes (see Figure 1(B), Figure 2).

\section{Discussion}

In the $1990 \mathrm{~s}$, there have been an increasing number of reports of short and long-term complications associated with angle-supported anterior chamber pIOLs including

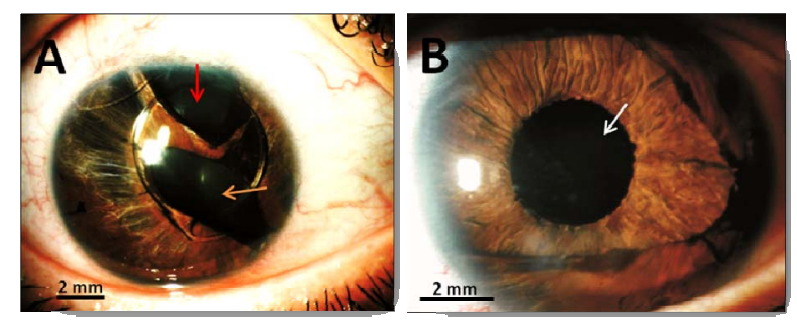

Figure 1. (A) Pupil ovalization (orange arrow) and large iris defect (red arrow) in the patient's left eye following implantation with an angle-supported phakic IOL (Morcher type 54); (B) The left eye two months postoperatively. The Dr. Schmidt Artificial iris has created a round pseudopupil (white arrow). 


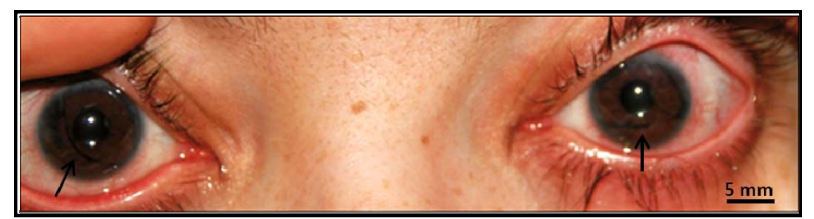

Figure 2. Appearance of the right and left eyes one year postoperatively. The Dr. Schmidt Artificial iris was positioned into the sulcus under the natural iris remnants (arrows). Note the excellent cosmetic match between the prosthesis and the color of the patient's native iris.

corneal endothelial cells loss, pupil ovalization, iris atrophy, glaucoma, and chronic anterior uveitis [7]. If today most early designs have been abandoned, surgeons still are facing late implant-related complications as reported here with the myopic Morcher type $54 \mathrm{pIOL}$. The patient was impaired by photophobia, glare, halo and loss in visual acuity and was seriously distressed due to the cosmetically deforming aspect of her eyes. The management of this case was challenging due to the poor preoperative status of the corneal endothelium that would be further affected by another surgery, the presence of glaucoma and cataract, and also because the iris defect was so severe that implantation of an iris prosthesis was necessary. Furthermore, besides all the functional aspects, aesthetic issues had to be considered. Thus, we selected the Dr. Schmidt iris prosthesis that could address both aspects. Our patient was managed by explantation of the pIOL combined with cataract extraction and simultaneous implantation of a standard IOL in the bag and an artificial iris in the sulcus.

One of the most attractive aspects of the Dr. Schmidt artificial iris is its flexibility and foldability that allow insertion through a sutureless small incision, thus minimizing potential surgically induced astigmatism. In our case though, because a large incision was required for the pIOL explantation, we used the same incision for implantation of the prosthesis and the standard IOL. The surgery was uneventful and no serious complications such as irritation, inflammation and corneal decompensation occurred during the one year postoperative period. In spite of the patient's preoperative advanced glaucoma, only one eye required an increase in antiglaucoma medications 10-month postoperatively. At the last visit, there was no significant change in the manifest cylinder and both eyes had reached UDVA of 0.3 and CDVA of 0.6 ; a very satisfactory outcome considering the patient preexisting ocular comorbidity. The patient also reported a significant improvement in the quality of vision due to the disappearance of photic disturbances.

The primary function of iris prostheses is to minimize visual disturbances by providing an artificial pupil which limits the amount of light entering the eye, to promote depth of focus and to limit spherical and chromatic aber- rations associated with large pupil sizes. The Dr. Schmidt artificial iris was designed with a small fixed aperture of $3.35 \mathrm{~mm}$, thereby reducing maximally the symptoms of glare and daytime photophobia. We have also found the size to be adequate for examination of the peripheral retina. In addition to significant visual disabilities, patients with iris defects usually suffer of psychological and social disabilities because of the disfigured appearance of their eyes. Besides its functional role, the Dr. Schmidt artificial iris offers the advantage of reproducing a patient's original iris appearance due to the flexibility of its silicone material and colour customization. Our patient was very pleased in the cosmetic appearance of her eyes and has returned to a normal social life.

In summary, despite our patient pre-existing ocular comorbidity, implantation of a Dr. Schmidt artificial iris proved to be a safe and effective method to improve visual quality and quality of life. This device represents a significant aesthetic improvement over other artificial iris devices that are currently available on the market.

\section{REFERENCES}

[1] S. E. Burk, A. P. Da Mata, M. E. Snyder, R. J. Cionni, J. S. Cohen and R. H. Osher, "Prosthetic Iris Implantation for Congenital, Traumatic, or Functional Iris Deficiencies," Journal of Cataract and Refractive Surgery, Vol. 27, No. 11, 2001, pp.1732-1740. doi:10.1016/S0886-3350(01)01124-5

[2] I. Mavrikakis and J. M. Casey, "Phacoemulsification and Endocapsular Implantation of An Artificial Iris Intraocular Lens in Traumatic Cataract and Aniridia," Journal of Cataract and Refractive Surgery, Vol. 28, No. 7, 2002, pp. 1088-1091. doi:10.1016/S0886-3350(02)01331-7

[3] G. Beltrame, M. L. Salvetat, M. Chizzolini, G. B. Driussi, P. Busatto, G. Di Giorgio, F. Barosco and B. Scuderi, "Implantation of A. Black Diaphragm Intraocular Lens in Ten Cases of Post-Traumatic Aniridia," European Journal of Ophthalmology, Vol. 13, No. 1, 2003, pp. 62-68.

[4] J. L. Menezo, R. Martínez-Costa, A. Cisneros and M. C. Desco, "Implantation of Iris Devices in Congenital and Traumatic Aniridias: Surgery Solutions and Complications," European Journal of Ophthalmology, Vol. 15, No. 4, 2005, pp. 451-457.

[5] N. A. Pozdeyeva, N. P. Pashtayev, V. P. Lukin and Y. N. Batkov, "Artificial Iris-Lens Diaphragm in Reconstructive Surgery for Aniridia and Aphakia," Journal of Cataract and Refractive Surgery, Vol. 31, No. 9, 2005, pp. 1750-1759. doi:10.1016/j.jcrs.2005.02.037

[6] R. S. Mashor, I. Bahar, I. Kaiserman, A. L. Berg, A. Slomovic and D. S. Rootman, "Combined Penetrating Keratoplasty and Implantation of Iris Prosthesis Intraocular Lenses after Ocular Trauma," Journal of Cataract and Refractive Surgery, Vol. 37, No. 3, 2011, pp. 582-587. doi:/10.1016/j.jcrs.2010.10.038

[7] T. Kohnen, D. Kook, M. Morral and J. L. Güell, "Phakic 
Intraocular Lenses: Part 2: Results and Complications," Journal of Cataract and Refractive Surgery, Vol. 36, No.
12, 2010, pp. 2168-2194.

doi:10.1016/j.jcrs.2010.10.007 